\title{
Efficacy and Safety of UI05MSP015CT in Functional Dyspepsia: A Randomized, Controlled Trial
}

Hyuk Yoon ${ }^{1}$, Dong Ho Lee ${ }^{1}$, Yong-Hyun Lee ${ }^{2}$, Ju-Cheol Jeong ${ }^{2}$, Soo Teik Lee ${ }^{3}$, Myung-Gyu Choi ${ }^{4}$, Seong Woo Jeon ${ }^{5}$, Ki-Nam Shim ${ }^{6}$, Gwang Ho Baik ${ }^{7}$, Jae Gyu Kim ${ }^{8}$, Jeong Seop Moon ${ }^{9}$, In-Kyung Sung ${ }^{10}$, Sang Kil Lee ${ }^{11}$, Poong-Lyul Rhee ${ }^{12}$, Hwoon-Yong Jung ${ }^{13}$, Bong Eun Lee ${ }^{14}$, Hyun Soo Kim ${ }^{15}$, Sang Gyun Kim ${ }^{16}$, Kee Myung Lee ${ }^{17}$, Jae Kyu Seong ${ }^{18}$, Jin Seok Jang ${ }^{19}$, and Jong-Jae Park ${ }^{20}$

\begin{abstract}
${ }^{1}$ Department of Internal Medicine, Seoul National University Bundang Hospital, Seongnam, ${ }^{2}$ Department of Clinical Research, Korea United Pharm. Inc., Seoul, ${ }^{3}$ Department of Internal Medicine, Chonbuk National University Hospital, Jeonju, ${ }^{4}$ Department of Internal Medicine, Seoul St. Mary's Hospital, The Catholic University of Korea, Seoul, ${ }^{5}$ Department of Internal Medicine, Kyungpook National University Medical Center, Daegu, ${ }^{6}$ Department of Internal Medicine, Medical Research Institute, Ewha Womans University School of Medicine, Seoul, ${ }^{7}$ Department of Internal Medicine, Hallym University Chuncheon Sacred Heart Hospital, Hallym University College of Medicine, Chuncheon, ${ }^{8}$ Department of Internal Medicine, Chung-Ang University College of Medicine, ${ }^{9}$ Department of Internal Medicine, Inje University Seoul Paik Hospital, Inje University College of Medicine, ${ }^{10}$ Department of Internal Medicine, Konkuk University College of Medicine, ${ }^{11}$ Department of Internal Medicine, Severance Hospital, Yonsei University College of Medicine, ${ }^{12}$ Department of Medicine, Samsung Medical Center, Sungkyunkwan University School of Medicine, ${ }^{13}$ Department of Internal Medicine, Asan Medical Center, Ulsan University College of Medicine, Seoul, ${ }^{14}$ Department of Gastroenterology, Pusan National University Hospital, Busan, ${ }^{15}$ Department of Internal Medicine, Chonnam National University Hospital, Gwangju, ${ }^{16}$ Department of Internal Medicine, Seoul National University Hospital, Seoul, ${ }^{17}$ Department of Gastroenterology, Ajou University School of Medicine, Suwon, ${ }^{18}$ Department of Internal Medicine, Chungnam National University Hospital, Daejeon, ${ }^{19}$ Department of Gastroenterology, Dong-A University Hospital, Busan, and ${ }^{20}$ Department of Internal Medicine, Korea University Guro Hospital, Seoul, Korea
\end{abstract}

Background/Aims: To evaluate the efficacy and safety of a controlled release, once-daily formulation of mosapride (UI05MSP015CT) in patients with functional dyspepsia (FD). Methods: Patients with FD were randomly assigned (1:1) to receive either UI05MSP015CT (15 mg once a day, study group) or mosapride (5 mg three times a day, control group) and corresponding placebo for 4 weeks. The primary endpoint was a change in the gastrointestinal symptom score (GIS) evaluated at enrollment and after 4 weeks. Secondary endpoints were changes in the Nepean Dyspepsia Index-Korean version (NDI-K), rate of satisfactory symptom relief, and rate of adverse events. Results: A total of 138 patients were enrolled (female, 73.9\%; mean age, 44.0 \pm 15.4 years). After excluding patients who violated the study protocol, 59 and 58 patients from the study and control groups, respectively, were included in the per-protocol analysis. No difference was observed in drug compliance between the control and study groups $(97.07 \% \pm 4.52 \%$ vs $96.85 \% \pm 6.05 \%, p=0.870)$. Changes in GIS scores were $-9.69 \pm 6.44$ and $-10.01 \pm 5.92$ in the study and control groups. The mean difference in GIS change between groups was 0.33 (95\% confidence interval, -1.75 to 2.41 ), demonstrating non-inferiority of UI05MSP015CT $(p=0.755)$. The rate of satisfactory symptom relief was not different between the study and control groups (39.0\% vs $56.9 \%, p=0.053$ ). No differences in change in NDI-K score (14.3 vs $16.9, p=0.263$ ) or rates of adverse events $(12.9 \%$ vs. $4.4 \%, p=0.062)$ were observed between the study and control groups. Conclusions: Once-daily mosapride is not inferior to conventional mosapride in efficacy and is safe in patients with FD. (Gut Liver 2018;12:516522)

Key Words: Mosapride; Functional dyspepsia; Compliance; Efficacy; Randomized clinical trial

\section{INTRODUCTION}

Mosapride is a selective 5-hydroxytryptamine $4\left(5-\mathrm{HT}_{4}\right)$ receptor agonist, which facilitates motility of smooth muscle in the gastrointestinal tract and increases gastric emptying by promoting the release of acetylcholine at nerve terminals in the mesenteric plexus. ${ }^{1,2}$ As mosapride has low affinity for $5-\mathrm{HT}_{1}$, 5- $\mathrm{HT}_{2}, \alpha_{1}, \alpha_{2}$, and $\mathrm{D}_{2}$ receptors in the gastrointestinal tract, it does not cause side effects such as arrhythmia, extrapyramidal symptoms, and hyperprolactinemia that are common for most prokinetics. ${ }^{3}$ Efficacy of mosapride in the treatment of func-

Correspondence to: Dong Ho Lee

Department of Internal Medicine, Seoul National University Bundang Hospital, 82 Gumi-ro 173beon-gil, Bundang-gu, Seongnam 13620, Korea

Tel: +82-31-787-7006, Fax: +82-31-787-4051, E-mail: dhljohn@yahoo.co.kr

Received on September 18, 2017. Revised on February 5, 2018. Accepted on March 29, 2018.

pISSN 1976-2283 eISSN 2005-1212 https://doi.org/10.5009/gnl17416

(a) This is an Open Access article distributed under the terms of the Creative Commons Attribution Non-Commercial License (http://creativecommons.org/licenses/by-nc/4.0) which permits unrestricted non-commercial use, distribution, and reproduction in any medium, provided the original work is properly cited. 
tional dyspepsia was demonstrated in many clinical studies. ${ }^{4-6}$ In addition to relieving symptoms, mosapride was also reported to improve quality of life of patients with functional dyspepsia. ${ }^{7}$

UI05MSP015CT (Gastiin CR ${ }^{\circledR}$, Korea United Pharm. Inc., Seoul, Korea) is a controlled release mosapride tablet, newly developed to increase patient convenience and compliance using sustained-release double-layer tablet formulation. Mosapride UI05MSP015CT formulation consists of two layers, providing immediate and sustained drug release. Immediate-release layer (mosapride citrate $5 \mathrm{mg}$ ) is instantly released and dissolves rapidly within 30 minutes of administration, whereas the sustainedrelease layer (mosapride citrate $10 \mathrm{mg}$ ) is a hydrophilic matrixtype tablet slowly dissolving over 24 hours. Pharmacokinetic profile of the formulation allows once-daily administration of UI05MSP015CT. In a phase I clinical trial in healthy male volunteers aged 20 to 55 years, UI05MSP015CT showed a pharmacokinetic profile similar to that of conventional mosapride. ${ }^{8}$

In this study, we performed a phase III clinical trial to demonstrate non-inferiority of UI05MSP015CT to mosapride in patients with functional dyspepsia.

\section{MATERIALS AND METHODS}

\section{Patients}

The inclusion criteria were as follows: (1) age >19 years; (2) diagnosis of functional dyspepsia meeting Rome III criteria (presenting at least one symptom among bothersome postprandial fullness, early satiation, epigastric pain or epigastric burning at least 6 months before the study, with $>3$ months duration) ${ }^{9}$ (3) at least three moderate or severe symptoms included in the gastrointestinal symptom score (GIS), ${ }^{10,11}$ with severity of 10 GIS items (nausea, vomiting, bloating, abdominal cramps, early satiety, acid eructation/heartburn, sickness, loss of appetite, retrosternal discomfort, and epigastric or upper abdominal pain) experienced in the last 2 weeks was evaluated by summing up scores from a 5-point Likert scale (0-4, no symptom, minor, moderate, severe, and extremely severe symptom, respectively); (4) no structural lesions of upper gastrointestinal tract detected endoscopically 12 weeks prior to enrollment. Although prokinetics is preferentially recommended for the treatment of functional dyspepsia of postprandial distress syndrome subtype, ${ }^{12}$ there is still a lack of qualified studies evaluating treatment response according to each subtype of functional dyspepsia using Rome criteria III. ${ }^{13}$ In addition, because mosapride is effective in epigastric pain syndrome subtype as well as postprandial distress syndrome subtype, ${ }^{4-6}$ we included patients with both subtypes of functional dyspepsia in this study. Similar to the previous study that evaluated prevalence and characteristics of functional dyspepsia using Rome III questionnaire in Korea, ${ }^{14}$ patients who had epigastric pain or burning but who did not completely satisfy epigastric pain syndrome criteria were classified as nonspecified type of functional dyspepsia. An additional criterion (nausea, vomiting, and acid eructation/heartburn present among GIS items) was added after Korea Food and Drug Administration recommended the evaluation of efficacy of UI05MSP015CT for approved indication of mosapride in Korea (improvement of these symptoms stemming from functional dyspepsia).

Exclusion criteria were as follows: (1) history of dyspepsia unrelated to functional dyspepsia or diseases that may affect evaluation of efficacy in the previous 6 months; (2) history of gastrointestinal surgery; (3) history of malignancy in the previous 5 years; (4) psychiatric disorders including major depressive disorder and anxiety; (5) liver cirrhosis or abnormal liver laboratory findings ( $>3$ times upper normal limit of aspartate or alanine aminotransferase levels); (6) advanced chronic kidney disease (creatinine clearance $<30 \mathrm{~mL} / \mathrm{min}$ ); (7) uncontrolled hypertension (systolic/diastolic blood pressure $\geq 160 / 100 \mathrm{mmHg}$ ); (8) uncontrolled diabetes (HbA1c $>8 \%$ ); (9) pregnancy and lactation; (10) recent history of taking medication affecting the gastrointestinal system: prokinetics, erythromycin, acid release inhibitors (histamine 2 receptor antagonists, proton pump inhibitors, or potassium-competitive acid blockers), gastric mucosa protectors, fundal relaxants (sumatriptan, buspirone), cholinergics, anticholinergics, antispasmodics, antidepressants (tricyclic antidepressants and selective serotonin reuptake inhibitors), aspirin over $100 \mathrm{mg} /$ day, systemic nonsteroidal anti-inflammatory drugs, and systemic glucocorticosteroids. Patients treated with any of the listed drugs who wished to participate in this study were eligible for enrollment after a wash-out period of 2 weeks.

\section{Study design}

This study was designed as a multicenter, randomized, activecontrolled, double-blind, non-inferiority, phase III clinical trial evaluating efficacy and safety of UI05MSP015CT in functional dyspepsia. After a 2-week wash-out period, patients with functional dyspepsia were randomly assigned (1:1) to receive either UI05MSP015CT (15 mg once daily before breakfast, study group) or mosapride (Gasmotin ${ }^{\circledR}$; Daewoong Pharmaceutical, Seoul, Korea) (5 mg three times a day before each meal, control group) and corresponding placebo three times a day or once a day for 4 weeks. This trial was registered at ClinicalTrials.Gov (NCT03225248). In addition, this study was approved by the Institutional Review Board of Seoul National University Bundang Hospital (IRB number: B-1407/260-005) and all other institutions. All patients provided informed consent.

All patients underwent a urea breath test before enrollment to ascertain Helicobacter pylori infection status. GIS was evaluated at enrollment, and after 2 and 4 weeks of treatment. Nepean Dyspepsia Index-Korean version (NDI-K) questionnaire was administered at enrollment and after 4 weeks of treatment. Drug compliance was determined by monitoring the unused amount of the clinical trial drug returned by the patients who visited the clinic of each hospital after 2 and 4 weeks of treatment. 
Primary endpoint was the change of total GIS score, evaluated at enrollment and after 4 weeks. Secondary endpoints were rate of satisfactory symptom relief, change in quality of life evaluated using NDI-K, and rate of adverse events. Symptom relief was assessed as follows. Patients were asked "How much has the dyspepsia symptom improved in the last 2 weeks compared to dyspepsia before treatment start?" and responded by selecting: "loss of symptom," “apparent improvement," "moderate improvement," "no change," and "deterioration." Rate of satisfactory symptom relief was defined as the proportion of enrolled patients who answered as "loss of symptom" or "apparent improvement." NDI-K consists of 25 questions on five quality of life areas: stress/sleep, disturbance of daily life, eating/ drinking, knowledge and control, and work/study. Questions are scored 1-5 points, with higher score indicating better quality of life. Importance of the five quality of life areas was weighed by the patients (scale of 1-5) and the associated question scores were multiplied by the weighing factors before summation to obtain area scores. Area scores were converted using the following formula: ([area score - minimum area score]/score range of each area) $\times 100$. Total NDI-K score was calculated by summing converted area scores.

Adverse events that occurred during the clinical trial were classified as treatment-emergent adverse events, adverse drug reactions, and serious adverse events. A treatment-emergent adverse event was defined as any event not present prior to the initiation of the treatments or any event already present that worsens in either intensity or frequency following exposure to the treatments. An adverse drug reaction was defined as any adverse event for which there is a reasonable possibility that the drug caused the adverse event; "reasonable possibility" means there is evidence to suggest a causal relationship between the drug and adverse event. An adverse event was considered "se- rious" if it results in any of the following outcomes: death, a life-threatening adverse event, inpatient hospitalization or prolongation of existing hospitalization, a persistent or significant incapacity or substantial disruption of the ability to conduct normal life functions, or a congenital anomaly/birth defect.

Enrolled patients receiving at least one clinical trial drug were included in intention-to-treat (ITT) analysis, whereas patients who completed the clinical trial without major protocol violations were included in per-protocol (PP) analysis. Major protocol violations included dropout, breach of inclusion/exclusion criteria, taking a prohibited drug, study drug compliance $<80 \%$, deviation from scheduled clinic visit date, and mistakes in treatment dispensation.

\section{Statistical analysis}

SPSS for Windows version 18.0 (SPSS Inc., Chicago, IL, USA) was used for the statistical analysis. Continuous variables were analyzed using Student t-test. The chi-square test or Fisher exact test were used to analyze categorical variables. Two-sided 95\% confidence interval (CI) was calculated for the difference in the change of GIS score after 4 weeks between the groups. In the studies that evaluated the efficacy of prokinetic agents similar to mosapride, the change of GIS score after 4 weeks in the study group and control group was -6.6 to -12.1 and -1.5 to -2.1 , respectively. ${ }^{10,11,15}$ We assumed that the change of GIS score in our study would be weighted-mean value of the results of the afore-mentioned studies. Therefore, we reasoned that 3.83 , which is the $50 \%$ of weighted-mean difference of study and control group $(-9.45--1.80=-7.65)$ would be the acceptable upper limit of non-inferiority. Finally, taking a more conservative stance, non-inferiority was declared if the upper limit of the CI was $\leq 3$. For sample size calculation, we set $\alpha$ error as 0.025 one-sided significance level and gave $80 \%$ power;

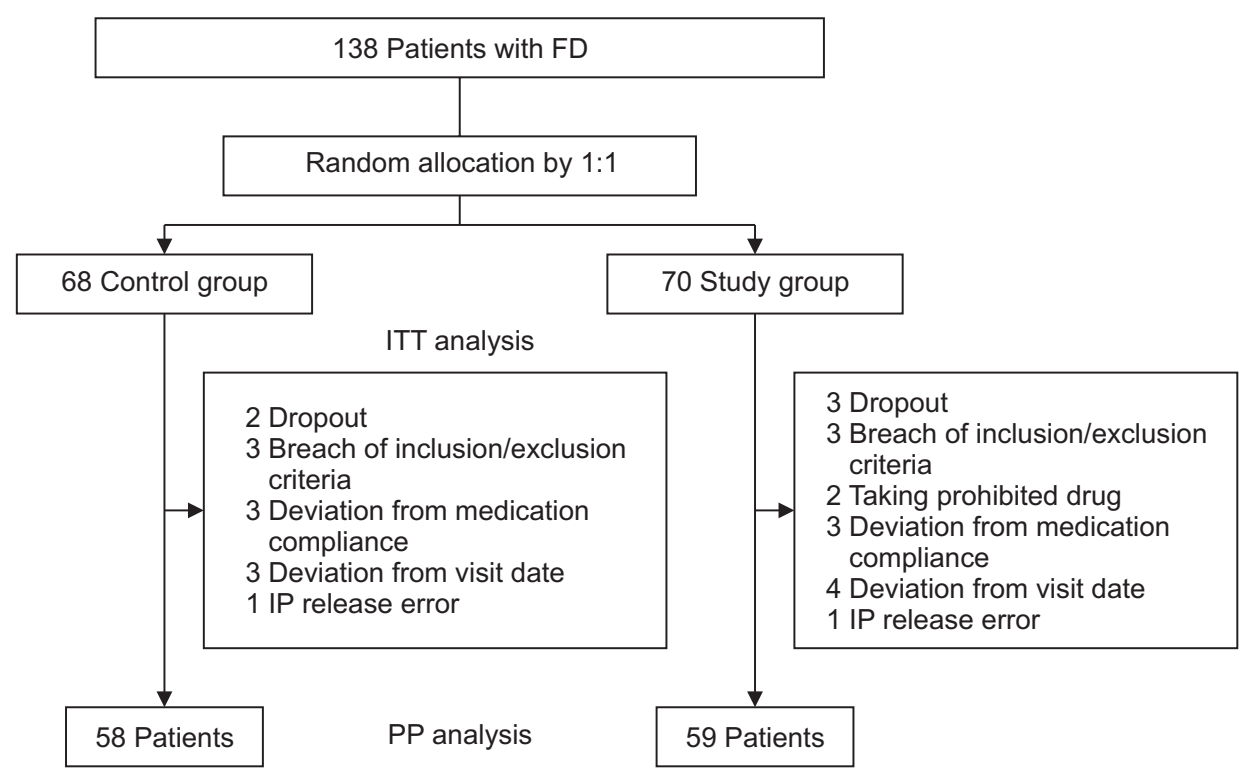

Fig. 1. Flow schematic of the study: intention-to-treat (ITT) and perprotocol (PP) analyses.

FD, functional dyspepsia; IP, investigational product. Some patients were excluded from the study for several reasons. 
true difference was assumed as zero. From these backgrounds, 52 subjects were required in each group (1:1 allocation). We estimated dropout rate as 30\% and finally we planned to enroll 75 subjects in each group (total 150 subjects). p-values $<0.05$ were consider statistically significant. Statistical methods of this study were reviewed by researcher (Hak-youn Kim) from C\&R Research, Inc.

\section{RESULTS}

Fig. 1 shows the flow schematic of the study (ITT and PP analyses). A total of 138 patients with functional dyspepsia (study group, 70; control group, 68) were enrolled between November 2014 and August 2015 in 19 university tertiary care hospitals in South Korea (ITT analysis set: female 73.9\%, mean age $44.0 \pm 15.4$ years). Because an interim analysis indicated sufficient statistical power for PP analysis, recruitment was stopped before randomization of 150 patients. After excluding patients who violated study protocol (see Materials and Methods), 59 and 58 patients from study and control groups, respectively, were included in PP analysis. Table 1 shows the baseline characteristics of both groups (ITT analysis). The only difference between control and study groups was body mass index $(22.03 \pm 3.51$ $\mathrm{kg} / \mathrm{m}^{2}$ vs $\left.23.41 \pm 3.73 \mathrm{~kg} / \mathrm{m}^{2}, \mathrm{p}=0.007\right)$. The proportion of postprandial distress syndrome subtype was higher in control group than in study group, there was no statistical significance (48.5\% vs $41.4 \%, p=0.207$ ). No difference was observed in drug compliance between control and study groups in PP analysis (97.07\% $\pm 4.52 \%$ vs $96.85 \% \pm 6.05 \%, p=0.870$ ).

Total GIS score decreased gradually in both groups during the study period (Fig. 2A). In PP analysis, mean change of GIS total score against baseline after 4 weeks was $-10.03 \pm 5.53$ and $-9.80 \pm 6.00$ in control and study groups, respectively (Fig. 2B). Difference in the change of GIS score between study and control groups was 0.24 (95\% CI, -1.88 to 2.35 ). As the upper limit of the CI was $<3$, study drug was not inferior to control drug $(p=0.824)$. No difference in the change of GIS score was observed between the groups for any of the symptoms after 4 weeks (Table 2). When we performed subgroup analysis in the patients with postprandial distress syndrome, mean change of GIS total score against baseline after 4 weeks was $-10.72 \pm 5.83$ and $-9.28 \pm 4.88$ in control and study groups, respectively. Difference in the change of GIS score between study and control

Table 1. Baseline Patient Characteristics (Intention-to-Treat Analysis)

\begin{tabular}{lccc}
\hline \multicolumn{1}{c}{ Characteristic } & $\begin{array}{c}\text { Control group } \\
(\mathrm{n}=68)\end{array}$ & $\begin{array}{c}\text { Study group } \\
(\mathrm{n}=70)\end{array}$ & $\mathrm{p}$-value \\
\hline Age, yr & $44.90 \pm 15.03$ & $43.73 \pm 15.85$ & 0.547 \\
Female sex & $52(76.5)$ & $50(71.4)$ & 0.444 \\
BMI, kg/m & $22.03 \pm 3.51$ & $23.41 \pm 3.73$ & $0.007^{*}$ \\
Smoking & & & 0.708 \\
Never & $57(83.8)$ & $62(88.6)$ & \\
Past & $5(7.4)$ & $4(5.7)$ & \\
Current & $6(8.8)$ & $4(5.7)$ & \\
Alcohol ${ }^{\dagger}$ & & & 0.412 \\
Never & $41(60.3)$ & $36(51.4)$ & \\
Past & $5(7.4)$ & $4(5.7)$ & \\
Current & $21(30.9)$ & $29(41.4)$ & \\
Disease duration, yr & $3.90 \pm 5.23$ & $4.06 \pm 5.83$ & 0.569 \\
Subtype of FD & & & 0.207 \\
PDS & $33(48.5)$ & $29(41.4)$ & \\
EPS & $1(1.5)$ & 0 & \\
Non-specified & $34(50.0)$ & $41(58.6)$ & \\
Helicobacter pylori infection & $29(42.6)$ & $27(38.6)$ & 0.586 \\
\hline Da & & & \\
\hline
\end{tabular}

Data are presented as mean \pm SD or number (\%).

BMI, body mass index; FD, functional dyspepsia; PDS, postprandial distress syndrome; EPS, epigastric pain syndrome.

${ }^{*} \mathrm{p}<0.05$; ${ }^{\dagger}$ There were some missing data.
A

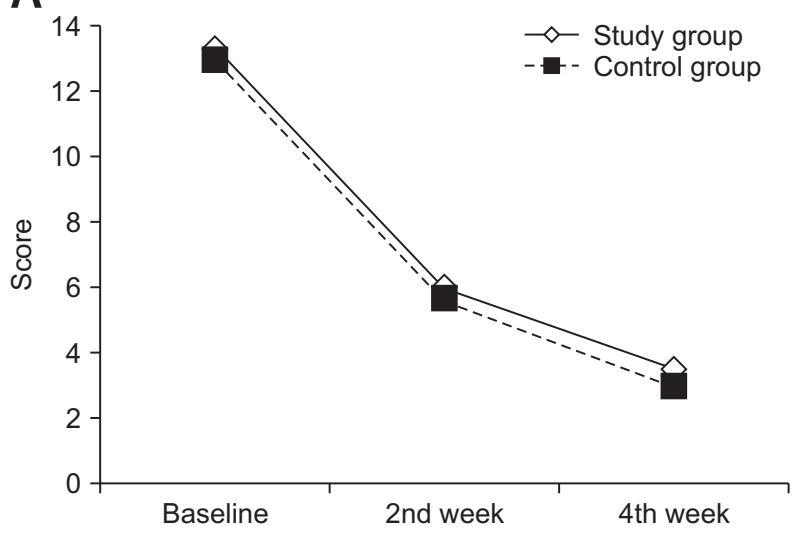

B

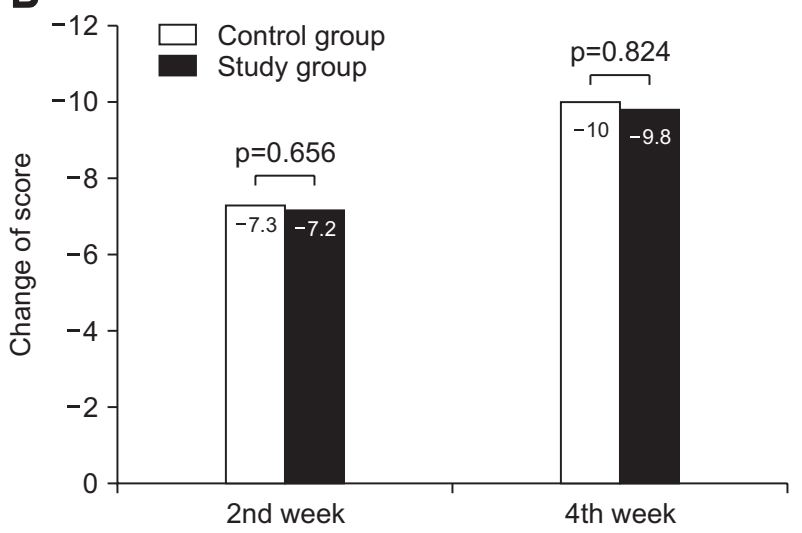

Fig. 2. Primary endpoint: GIS total score. (A) GIS total score by visit. (B) Change in GIS total score compared to baseline by visit. GIS, gastrointestinal symptom score. 
Table 2. Change in GIS Score by Symptoms after 4 Weeks (PerProtocol Analysis)

\begin{tabular}{lccc}
\hline \multicolumn{1}{c}{ Symptoms } & $\begin{array}{c}\text { Control } \\
(\mathrm{n}=58)\end{array}$ & $\begin{array}{c}\text { Study } \\
(\mathrm{n}=59)\end{array}$ & $\mathrm{p}$-value \\
\hline Nausea & $-1.19 \pm 1.02$ & $-1.19 \pm 1.24$ & 0.765 \\
Vomiting & $-0.48 \pm 0.94$ & $-0.63 \pm 1.05$ & 0.432 \\
Bloating & $-1.53 \pm 0.92$ & $-1.44 \pm 0.91$ & 0.542 \\
Abdominal cramps & $-0.55 \pm 0.88$ & $-0.63 \pm 1.02$ & 0.849 \\
Early satiety & $-1.45 \pm 0.92$ & $-1.25 \pm 1.18$ & 0.308 \\
Acid eructation/heartburn & $-1.50 \pm 1.01$ & $-1.69 \pm 1.00$ & 0.501 \\
Sickness & $-0.97 \pm 0.99$ & $-0.80 \pm 0.92$ & 0.332 \\
Loss of appetite & $-0.81 \pm 0.93$ & $-0.47 \pm 1.02$ & 0.090 \\
Retrosternal discomfort & $-0.69 \pm 0.99$ & $-0.73 \pm 0.94$ & 0.832 \\
Epigastric or upper abdominal pain & $-0.86 \pm 1.05$ & $-0.97 \pm 1.08$ & 0.502 \\
\hline
\end{tabular}

Data are presented as mean \pm SD.

GIS, gastrointestinal symptom score.

Table 3. Degree of Symptom Relief after 4 Weeks (Per-Protocol Analysis)

\begin{tabular}{lccc}
\hline & $\begin{array}{c}\text { Control group } \\
(\mathrm{n}=58)\end{array}$ & $\begin{array}{c}\text { Study group } \\
(\mathrm{n}=59)\end{array}$ & $\mathrm{p}$-value \\
\hline Loss of symptom & $9(15.52)$ & $5(8.48)$ & - \\
Apparent improvement & $24(41.38)$ & $18(30.51)$ & - \\
Moderate improvement & $20(34.48)$ & $30(50.85)$ & - \\
No change & $4(6.90)$ & $5(8.48)$ & - \\
Deterioration & $1(1.72)$ & $1(1.70)$ & - \\
Satisfactory symptom relief* & $33(56.9)$ & $23(39.0)$ & 0.053 \\
\hline
\end{tabular}

Data are presented as number (\%).

*Satisfactory symptom relief was defined as loss of a symptom or apparent improvement.

groups was 1.44 (95\% CI, -1.52 to 4.41$)$; there was no statistical significance $(\mathrm{p}=0.333)$.

Tables 3 and 4 show the degree of symptom relief and change of NDI-K score, respectively, after 4 weeks in both groups in PP analysis. The rate of satisfactory symptom relief was different between control and study groups in ITT analysis (55.9\% vs $37.1 \%, \mathrm{p}=0.027)$. However, it was not different between both groups in PP analysis (56.9\% vs 39.0\%, p=0.053). No differences in change of total NDI-K score were observed in both ITT and PP analysis (ITT: $16.68 \pm 14.15$ vs $13.79 \pm 12.23, \mathrm{p}=0.204$, PP: $16.91 \pm 13.44$ vs $14.30 \pm 11.62, p=0.263)$.

Table 5 shows treatment-emergent adverse events that occurred during the clinical trial. No difference in the total number of patients with adverse events was observed between control and study groups (4.4\% vs 12.9\%, p=0.062). Gastrointestinal disorders were the most common adverse events in the study group (one case each of diarrhea, frequent bowel movements, and nausea, 3/10=30.0\%). Diarrhea and nausea, among treatment-emergent adverse events in the study group, and no events in the control group were classified as adverse drug reac-
Table 4. Change in NDI-K Score after 4 Weeks (Per-Protocol Analysis)

\begin{tabular}{lccc}
\hline \multicolumn{1}{c}{ Items } & $\begin{array}{c}\text { Control } \\
(\mathrm{n}=58)\end{array}$ & $\begin{array}{c}\text { Study } \\
(\mathrm{n}=59)\end{array}$ & p-value \\
\hline Total score & $16.91 \pm 13.44$ & $14.30 \pm 11.62$ & 0.263 \\
$\quad$ Stress/sleep & $17.61 \pm 17.15$ & $12.92 \pm 14.80$ & 0.116 \\
Disturbance of daily life & $15.64 \pm 15.75$ & $13.66 \pm 15.25$ & 0.490 \\
Eating/drinking & $17.15 \pm 18.80$ & $13.94 \pm 16.46$ & 0.328 \\
Knowledge and control & $19.63 \pm 18.42$ & $16.70 \pm 19.42$ & 0.405 \\
Work/study & $14.51 \pm 16.55$ & $14.27 \pm 16.36$ & 0.936 \\
\hline
\end{tabular}

Data are presented as mean \pm SD.

NDI-K, Nepean Dyspepsia Index-Korean version.

Table 5. Treatment-Emergent Adverse Events (Intention-to-Treat Analysis)

\begin{tabular}{lccc}
\hline & $\begin{array}{c}\text { Control group } \\
(\mathrm{n}=68)\end{array}$ & $\begin{array}{c}\text { Study group } \\
(\mathrm{n}=70)\end{array}$ & p-value \\
\hline Abdominal discomfort & $1(1.5)$ & - & - \\
Diarrhea & - & $1(1.4)$ & - \\
Frequent bowel movements & - & $1(1.4)$ & - \\
Nausea & - & $1(1.4)$ & - \\
Depression & - & $1(1.4)$ & - \\
Psychogenic pain disorder & - & $1(1.4)$ & - \\
Positional vertigo & - & $1(1.4)$ & - \\
Bronchitis & - & $1(1.4)$ & - \\
Pyuria & $1(1.5)$ & - & - \\
Dyslipidemia & - & $1(1.4)$ & - \\
Urinary stone & - & $1(1.4)$ & - \\
Cough & - & $1(1.4)$ & - \\
Pruritus & $1(1.5)$ & - & - \\
Total & $3(4.4)$ & $9(12.9)^{*}$ & 0.062 \\
\hline
\end{tabular}

Data are presented as number (\%).

*Because depression and psychogenic pain disorder occurred in the same subject, the total number was decreased by one.

tions $(2.94 \%$ vs $0 \%, p=0.241)$. Among 10 adverse events in the study group, seven were mild and three were moderate, whereas all adverse events in the control group were mild. No serious adverse events were reported in either group.

\section{DISCUSSION}

We conducted this study applying similar disease definition, inclusion/exclusion criteria, and treatment duration as previous studies that evaluated drug efficacy in functional dyspepsia. ${ }^{10,11,15}$ As a result, demographics of enrolled patients such as mean age and sex distribution were also similar to previous studies. ${ }^{10,11,15}$ Additionally, because the baseline GIS score in both groups was similar to that of previous studies, ${ }^{10,11,15}$ we could confirm that the severity of functional dyspepsia in patients enrolled in this 
study was also comparable to previous results.

In the present study, the degree of symptom improvement of functional dyspepsia in the study group, evaluated by the change of total GIS score after 4 weeks of treatment, was not inferior to that in control group. In addition, no difference in the change of specific GIS scores, rate of satisfactory symptom relief, and quality of life was observed between the two groups, indicating that efficacy of UI05MSP015CT was comparable to mosapride in this study. As the rate of adverse drug reactions was $2.94 \%$ in the study group and no serious adverse events were reported, safety of UI05MSP015CT was considered acceptable.

Drug compliance is very important for successful treatment of many diseases, including functional dyspepsia. As the effects of functional dyspepsia drug treatments have been estimated to offer approximately 10\% improvement over placebo in many clinical trials, ${ }^{16}$ lack of patient adherence may render the treatment benefits marginal in clinical practice, leading to manifestation of adverse events without definite improvement of symptoms. One method to increase drug compliance is reducing dose frequency. ${ }^{17}$ For this reason, administering drugs once a day is recommended in the treatment of some chronic diseases such as ulcerative colitis. ${ }^{18}$ Mosapride is a safe and effective drug for treating functional dyspepsia. However, taking mosapride three times a day, 30 minutes before each meal to maximize treatment efficacy, makes the dosing regimen difficult for patients to adhere to. Whereas drug compliance is also important for short treatment-course ( 1 to 2 weeks) diseases such as $H$. pylori infection, ${ }^{19}$ the chronic nature of functional dyspepsia requires patients to comply with treatment over several months. Therefore, UI05MSP015CT, offering once a day dosing regimen, would be an excellent alternative to conventional mosapride in patients with functional dyspepsia. In this study, drug compliance was very good in both groups (>95\%). However, if this study had been performed without including corresponding placebo in each group, compliance would be higher in the study group than in control group.

It has been suggested that $H$. pylori may play a role in pathogenesis of functional dyspepsia, ${ }^{20}$ with $H$. pylori eradication effectively improving symptoms in some patients. ${ }^{21}$ Therefore, we performed subgroup analysis to elucidate whether the efficacy of UI05MSP015CT differed based on H. pylori infection status. UI05MSP015CT was not inferior in efficacy to conventional mosapride irrespective of $H$. pylori infection status (data not shown).

This study has several limitations. First, mean body mass index of the patients in the study group was significantly higher than that of the patients in control group. Body mass index affects pharmacokinetics and pharmacodynamics of drugs, an effect that varies greatly depending on the type of the drug. ${ }^{22}$ As we did not evaluate drug serum levels during this study, we cannot completely rule out the possibility that the differ- ence in body mass index between groups may bias the efficacy comparison of control and study drugs. Second, efficacy of the study drug could vary between different subtypes of functional dyspepsia. ${ }^{23}$ Because the number of patients who completely satisfied criteria for epigastric pain syndrome was too small, we could not perform subgroup analysis in the patients with this subtype of functional dyspepsia. Third, although most results of study endpoints showed similar trends in ITT and PP analysis, the rate of satisfactory symptom relief was different. It was lower in study group than in control group in ITT analysis. We could not adequately explain the cause of this finding. Finally, as UI05MSP015CT is formulated as a controlled release tablet that should be swallowed whole, not broken, ground up or chewed apart, this drug is not suitable for patients who require tube feeding.

In conclusion, administration of UI05MSP015CT to patients with functional dyspepsia for 4 weeks demonstrated non-inferiority of this formulation to conventional mosapride in efficacy and a comparable safety profile. Therefore, UI05MSP015CT offers a good alternative to conventional mosapride in patients with functional dyspepsia.

\section{CONFLICTS OF INTEREST}

This study was funded by Korea United Pharm. Inc.

\section{REFERENCES}

1. Pan H, Galligan JJ. 5-HT1A and 5-HT4 receptors mediate inhibition and facilitation of fast synaptic transmission in enteric neurons. Am J Physiol 1994;266(2 Pt 1):G230-G238.

2. Tonini M, Galligan JJ, North RA. Effects of cisapride on cholinergic neurotransmission and propulsive motility in the guinea pig ileum. Gastroenterology 1989;96(5 Pt 1):1257-1264.

3. Curran MP, Robinson DM. Mosapride in gastrointestinal disorders. Drugs 2008;68:981-991.

4. Hongo M, Harasawa S, Mine T, et al. Large-scale randomized clinical study on functional dyspepsia treatment with mosapride or teprenone: Japan Mosapride Mega-Study (JMMS). J Gastroenterol Hepatol 2012;27:62-68.

5. Kinoshita Y, Hashimoto T, Kawamura A, et al. Effects of famotidine, mosapride and tandospirone for treatment of functional dyspepsia. Aliment Pharmacol Ther 2005;21 Suppl 2:37-41.

6. Otaka M, Jin M, Odashima M, et al. New strategy of therapy for functional dyspepsia using famotidine, mosapride and amitriptyline. Aliment Pharmacol Ther 2005;21 Suppl 2:42-46.

7. Cho YK, Choi MG, Kim SH, et al. The effect of mosapride on quality of life in functional dyspepsia. Korean J Gastroenterol 2004;43:160-167.

8. Hong J. A phase 1, randomized, open-label, single dose, 3-treatment, 3-period Williams-design to assess the pharmacokinetic characteristics and food effect of mosapride citrate controlled- 
release tablets compared with mosapride citrate immediate-release tablets in healthy male subjects: clinical trial report. Seoul: Korea United Pharm, 2013:1-48.

9. Drossman DA. The functional gastrointestinal disorders and the Rome III process. Gastroenterology 2006;130:1377-1390.

10. Rösch W, Vinson B, Sassin I. A randomised clinical trial comparing the efficacy of a herbal preparation STW 5 with the prokinetic drug cisapride in patients with dysmotility type of functional dyspepsia. Z Gastroenterol 2002;40:401-408.

11. Madisch A, Holtmann G, Mayr G, Vinson B, Hotz J. Treatment of functional dyspepsia with a herbal preparation: a doubleblind, randomized, placebo-controlled, multicenter trial. Digestion 2004;69:45-52.

12. Camilleri M, Stanghellini V. Current management strategies and emerging treatments for functional dyspepsia. Nat Rev Gastroenterol Hepatol 2013;10:187-194.

13. Park JK, Huh KC, Shin CM, et al. Current issues in functional dyspepsia. Korean J Gastroenterol 2014;64:133-141.

14. Park JM, Choi MG, Cho YK, et al. Functional gastrointestinal disorders diagnosed by Rome III questionnaire in Korea. J Neurogastroenterol Motil 2011;17:279-286.

15. Madisch A, Melderis H, Mayr G, Sassin I, Hotz J. A plant extract and its modified preparation in functional dyspepsia: results of a double-blind placebo controlled comparative study. Z Gastroen- terol 2001;39:511-517.

16. Matsueda K, Hongo M, Tack J, Saito Y, Kato H. A placebo-controlled trial of acotiamide for meal-related symptoms of functional dyspepsia. Gut 2012;61:821-828.

17. Paes AH, Bakker A, Soe-Agnie CJ. Impact of dosage frequency on patient compliance. Diabetes Care 1997;20:1512-1517.

18. Choi CH, Moon W, Kim YS, et al. Second Korean guidelines for the management of ulcerative colitis. Intest Res 2017;15:7-37.

19. Malfertheiner P, Megraud F, O'Morain CA, et al. Management of Helicobacter pylori infection: the Maastricht IV/ Florence consensus report. Gut 2012;61:646-664.

20. Miwa H, Ghoshal UC, Gonlachanvit S, et al. Asian consensus report on functional dyspepsia. J Neurogastroenterol Motil 2012;18:150-168.

21. Du LJ, Chen BR, Kim JJ, Kim S, Shen JH, Dai N. Helicobacter pylori eradication therapy for functional dyspepsia: systematic review and meta-analysis. World J Gastroenterol 2016;22:34863495.

22. Hanley MJ, Abernethy DR, Greenblatt DJ. Effect of obesity on the pharmacokinetics of drugs in humans. Clin Pharmacokinet 2010;49:71-87.

23. Talley NJ, Ford AC. Functional dyspepsia. N Engl J Med 2015;373:1853-1863. 